\title{
Article \\ Immunomodulatory Properties of Umbilical Cord Blood-Derived Small Extracellular Vesicles and Their Therapeutic Potential for Inflammatory Skin Disorders
}

\author{
Sílvia C. Rodrigues ${ }^{1,2}{ }^{\oplus}$, Renato M. S. Cardoso ${ }^{1}$, Patricia C. Freire ${ }^{1}$, Cláudia F. Gomes ${ }^{1}$, Filipe V. Duarte ${ }^{1}{ }^{\circledR}$, \\ Ricardo Pires das Neves ${ }^{1,3,4}$ and Joana Simões-Correia $1,3,4, *$ (D) \\ 1 Exogenus Therapeutics, S.A., 3060-197 Cantanhede, Portugal; silviacouto25@gmail.com (S.C.R.); \\ renatocardoso83@gmail.com (R.M.S.C.); patricia.freire@exogenus-t.com (P.C.F.); \\ claudiafgomes.94@gmail.com (C.F.G.); filipevalenteduarte@gmail.com (F.V.D.); \\ ricardo.neves@uc-biotech.pt (R.P.d.N.) \\ 2 Doctoral Programme in Experimental Biology and Biomedicine (PDBEB), CNC-Center for Neuroscience and \\ Cell Biology, University of Coimbra, 3004-517 Coimbra, Portugal \\ 3 CNC - Center for Neuroscience and Cell Biology, CIBB - Centre for Innovative Biomedicine and \\ Biotechnology, University of Coimbra, 3004-517 Coimbra, Portugal \\ 4 IIIUC-Institute of Interdisciplinary Research, University of Coimbra, 3030-789 Coimbra, Portugal \\ * Correspondence: joana.correia@exogenus-t.com
}

Citation: Rodrigues, S.C.; Cardoso, R.M.S.; Freire, P.C.; Gomes, C.F.; Duarte, F.V.; Neves, R.P.d.; Simões-Correia, J.

Immunomodulatory Properties of Umbilical Cord Blood-Derived Small Extracellular Vesicles and Their Therapeutic Potential for Inflammatory Skin Disorders. Int. J. Mol. Sci. 2021, 22, 9797. https:// doi.org/10.3390/ijms22189797

Academic Editor: Terrence Piva

Received: 6 August 2021

Accepted: 6 September 2021

Published: 10 September 2021

Publisher's Note: MDPI stays neutral with regard to jurisdictional claims in published maps and institutional affiliations.

Copyright: (c) 2021 by the authors. Licensee MDPI, Basel, Switzerland. This article is an open access article distributed under the terms and conditions of the Creative Commons Attribution (CC BY) license (https:// creativecommons.org/licenses/by/ $4.0 /)$.

\begin{abstract}
Umbilical cord blood (UCB) has long been seen as a rich source of naïve cells with strong regenerative potential, likely mediated by paracrine signals. More recently, small extracellular vesicles (sEV), such as exosomes, have been shown to play essential roles in cell-to-cell communication, via the transport of numerous molecules, including small RNAs. Often explored for their potential as biomarkers, sEV are now known to have regenerative and immunomodulating characteristics, particularly if isolated from stem cell-rich tissues. In this study, we aim to characterize the immunomodulating properties of umbilical cord blood mononuclear cell-derived sEV (UCB-MNC-sEV) and explore their therapeutic potential for inflammatory skin diseases. UCB-MNC-sEV were shown to shift macrophages toward an anti-inflammatory phenotype, which in turn exert paracrine effects on fibroblasts, despite previous inflammatory stimuli. Additionally, the incubation of PBMC with UCB-MNC-sEV resulted in a reduction of total $\mathrm{CD}^{+}{ }^{+}$and $\mathrm{CD} 8^{+} \mathrm{T}$-cell proliferation and cytokine release, while specifically supporting the development of regulatory T-cells (Treg), by influencing FOXP3 expression. In a 3D model of psoriatic skin, UCB-MNC-sEV reduced the expression of inflammatory and psoriatic markers IL6, IL8, CXCL10, COX2, S100A7, and DEFB4. In vivo, UCB-MNC-sEV significantly prevented or reversed acanthosis in imiquimod-induced psoriasis, and tendentially increased the number of Treg in skin, without having an overall impact on disease burden. This work provides evidence for the anti-inflammatory and tolerogenic effect of UCB-MNC-sEV, which may be harnessed for the treatment of Th17-driven inflammatory skin diseases, such as psoriasis.
\end{abstract}

Keywords: extracellular vesicles; EV; umbilical cord blood; inflammatory skin disease; psoriasis

\section{Introduction}

Virtually every living cell releases extracellular vesicles (EV), which can be classified based on size and marker expression. One of the smallest known groups of EV, exosomes, have a diameter typically ranging from 30 to $100 \mathrm{~nm}$ and originate from endosomes [1]. Composed of lipids, proteins and nucleic acids, these ubiquitous vesicles are thought to be involved in multiple diseases, including inflammatory and autoimmune skin conditions [2]. Due to their physical characteristics, which allow them to carry molecules across long distances, EV are often explored for their potential as biomarkers [3,4]. Physiologically, small EV (sEV), such as exosomes, are key mediators of cellular communication, namely 
through microRNAs [5]. Hence, depending on the producing cell, sEV may have modulating characteristics, which can potentially be harnessed for therapeutic purposes. In fact, these naturally-produced vesicles are currently explored for the treatment of several conditions, including wound healing [6] and autoimmune diseases [7-9]. Their use replaces cell therapies $[10,11]$, while conferring advantages, namely concerning handling and formulation.

Chronic inflammatory skin disorders, such as psoriasis, represent a significant medical, psychological and financial burden to patients and healthcare systems [12,13]. Plaque psoriasis is characterized by patches of red, dry, and scaly skin, which often appear on the elbows, knees, and scalp. IL-17A and its upstream regulator IL-23 are two key molecules in the pathogenesis of psoriasis, and approaches that specifically target this pathway showed great clinical responses [14]. Despite these recent encouraging advances, continuing innovation is key for the development of new therapeutic approaches for patients who still have unmet needs, namely an incomplete treatment response, contraindications, or even affordability and practicality [15].

Umbilical cord blood (UCB) is a rich source of stem cells and immature T-cells [16] with potent suppressive ability [17]. The collection of UCB, commonly seen as medical waste, is non-invasive and has limited ethical concerns. We have previously shown that sEV from UCB mononuclear cells (UCB-MNC-sEV), produced using an established optimized protocol [18], accelerate the healing of diabetic wounds [6] and have a good safety profile [19]. In this study, we characterize the immunomodulating properties of UCB-MNC-sEV, and explore their potential in the treatment of psoriasis symptoms.

\section{Results}

\subsection{UCB-MNC-sEV Have Anti-Inflammatory and Tolerogenic Effects, Modulating Different} Immune Players Directly and Indirectly

While exploring the therapeutic potential of UCB-MNC-sEV for chronic wound healing $[6,18]$, we found differences in the immunological profile of treated versus control skin. Namely, 194 genes associated with immune system processes or inflammatory responses, corresponding to about $16 \%$ of all measured genes, were differentially expressed between the two groups of animals (Figure S1). Based on these results and on existing literature, we performed a series of in vitro proof-of-concept experiments to determine the nature and strength of UCB-MNC-sEV's immunomodulatory effect.

In line with studies using sEV from different sources [20,21], UCB-MNC-sEV promote the differentiation of macrophages into an anti-inflammatory M2, rather than a pro-inflammatory M1 phenotype (Figure 1). This effect is present both in unstimulated (Figure $1 \mathrm{a}-\mathrm{c}$ ) and LPS-stimulated macrophages (Figure 1d-f) and correlates with the de novo synthesis and release of inflammatory mediators (Figure 1a-1). Specifically, UCBMNC-sEV administration significantly decreases the level of IFNG, IL1B, PTSG2, and TNFA mRNA in macrophages, as well as the release of TNF $\alpha$ and CCL20 proteins. These data strongly indicate that UCB-MNC-sEV play a direct role in macrophage regulation, having an anti-inflammatory effect, even in the context of an acute pro-inflammatory stimulus.

At the local level, UCB-MNC-sEV directly promote the proliferation of dermal fibroblasts (Figure $1 \mathrm{~m}, \mathrm{n}$ ). Interestingly, the incubation of fibroblasts with conditioned medium from UCB-MNC-sEV-stimulated macrophages likewise modulates cell proliferation, as well as chemokine synthesis, with a more pronounced effect when compared to direct UCB-MNC-sEV stimulation (Figure 1o). These results suggest that, while UCB-MNC-sEV do act directly on target skin cells, their effects are likely reinforced by indirect mechanisms, depending on neighboring immune cell populations, such as macrophages. 

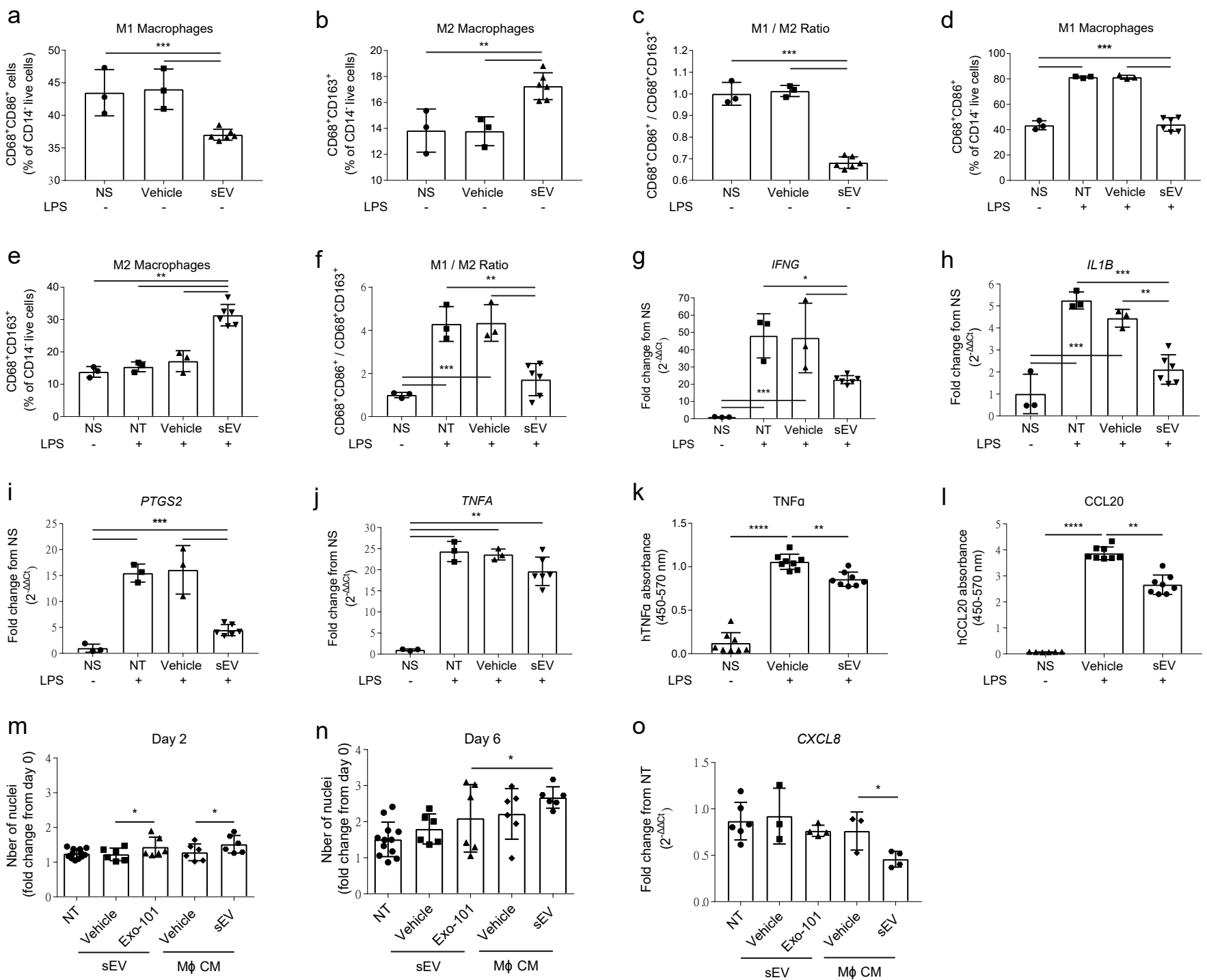

Figure 1. UCB-MNC-sEV's immunomodulatory effects in vitro. (a-f) THP-1-derived macrophages, with or without LPS stimulation as indicated, were incubated with $1 \times 10^{10}$ particles/mL of UCB-MNC-sEV for $24 \mathrm{~h}$, before flow cytometry analysis $(n \geq 3)$. (g-j) Relative expression of M1-associated genes by qPCR $(n \geq 3)$ and (k,1) release of pro-inflammatory molecules by ELISA $(n \geq 6)$. $(\mathbf{m}, \mathbf{n})$ Proliferation of normal human dermal fibroblasts, 2 and 6 days after incubation with UCB-MNC-sEV $\left(1 \times 10^{10}\right.$ particles $\left./ \mathrm{mL}\right)$ or media from UCB-MNC-sEV-stimulated macrophages $(n \geq 6)$, and (o) CXCL8 expression at $48 \mathrm{~h}(n \geq 3)$. All results are presented as mean $\pm \mathrm{SD}{ }^{*} p \leq 0.05,{ }^{* *} p \leq 0.01,{ }^{* * *} p \leq 0.001,{ }^{* * * *} p \leq 0.0001$. NS, non-stimulated; NT, non-treated; CM, conditioned media.

To evaluate the response of T-cells to UCB-MNC-sEV stimulation, in a physiological context, whole human PBMC were incubated for 6 days with a single dose of $1 \times 10^{10}$ vesicles $/ \mathrm{mL}$, following activation with $\alpha$-CD3/-CD28. UCB-MNC-sEV significantly reduced the proliferation of total, $\mathrm{CD} 4^{+}$and $\mathrm{CD} 8^{+} \mathrm{T}$-cells, as well as the intracellular content of IFN $\gamma$ in each of these populations (Figure 2a-f). The release of TNF $\alpha$ and CCL20 by total PBMCs was also significantly decreased (Figure 2g,h). Notably, after two days of treatment, UCB-MNC-sEV modulated the expression of population-specific transcription factors in $\mathrm{CD}^{+}$T-cells, with a trend toward the reduction of GATA-3, a significant decrease in T-bet and ROR $\gamma \mathrm{t}$, and a significant increase in Foxp3 mRNA (Figure 2i-1). This effect was still visible after 6 days of treatment, for ROR $\gamma \mathrm{t}$ and Foxp3 expression. 
a

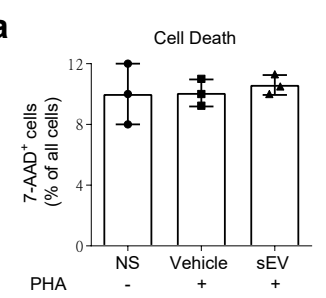

b

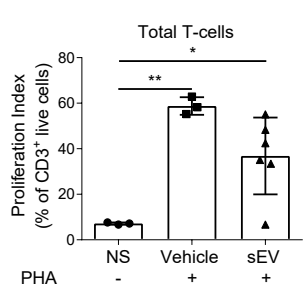

c

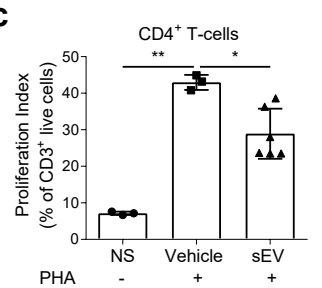

d

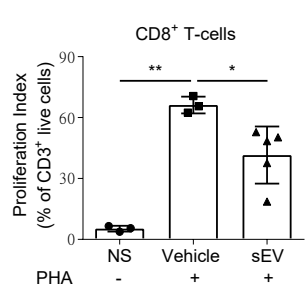

e

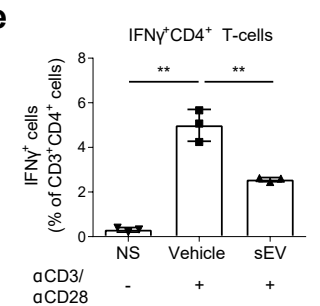

f

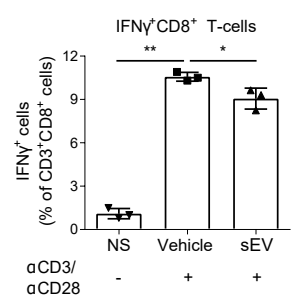

g

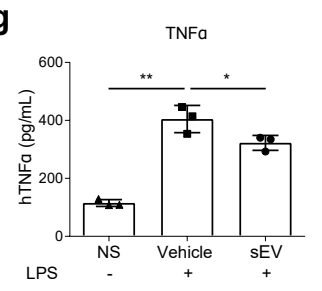

h

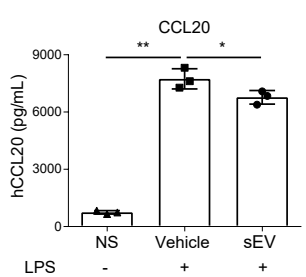

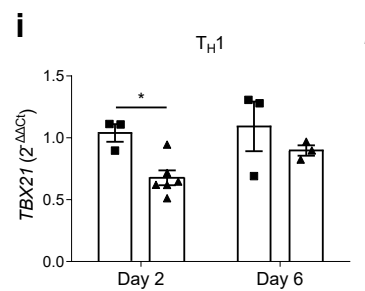
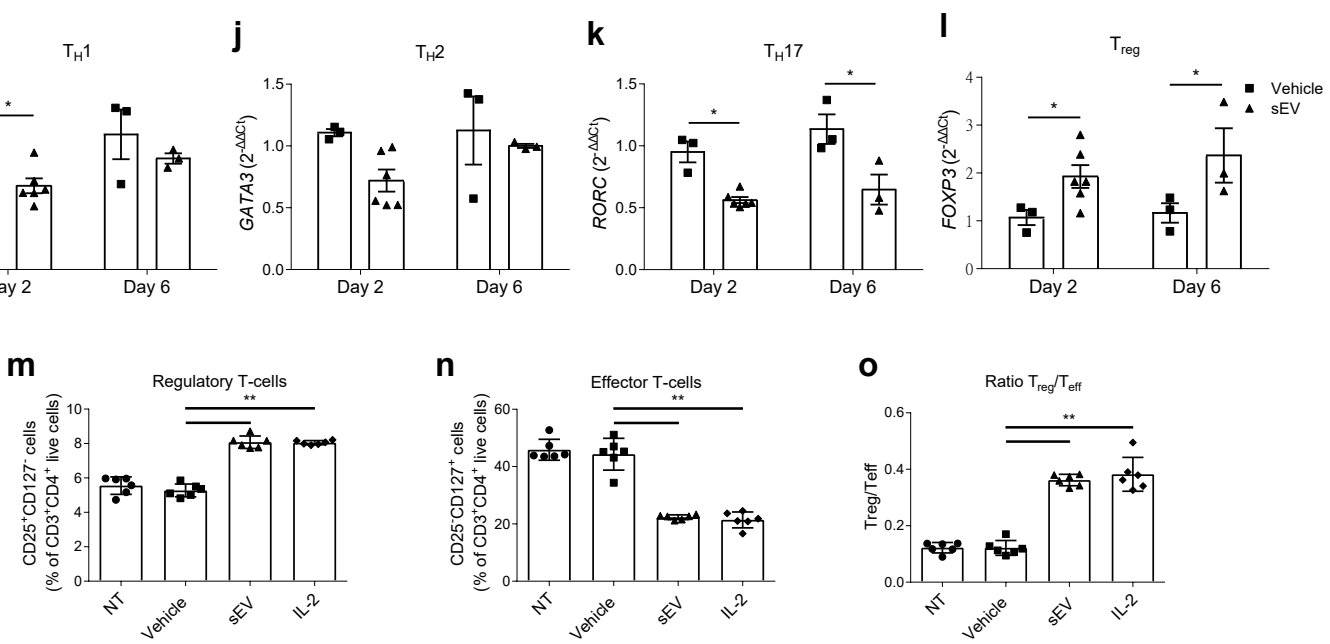

Figure 2. UCB-MNC-sEV's immunomodulatory effects on T-cells in vitro. (a-f) Human PBMC, stimulated with PMA or $\alpha \mathrm{CD} 3 / \alpha \mathrm{CD} 28$ as indicated, were incubated with a single dose of UCB-MNC-sEV at $1 \times 10^{10}$ particles $/ \mathrm{mL}$ for 6 days, followed by flow cytometry analysis $(n \geq 3)$. (g,h) TNF $\alpha$ and CCL20 release by LPS-stimulated PBMC after a $24 \mathrm{~h}$ incubation with $1 \times 10^{10}$ particles/mL UCB-MNC-sEV $(n=3)$. (i-1) Relative expression of transcription factors by PBMC incubated with UCB-MNC-sEV for 2 or 6 days ( $n \geq 3$ ). Cells were sorted based on FSC, SSC, CD3, and CD4, prior to RNA extraction. (m-o) Phenotyping of $\alpha \mathrm{CD} 3 / \alpha \mathrm{CD} 28$-activated PBMC, after 6 days of incubation with UCB-MNC-sEV at $1 \times 10^{10}$ particles $/ \mathrm{mL}$ or IL-2 $(100 \mathrm{IU} / \mathrm{mL})$ and TGF- $\beta(5 \mathrm{ng} / \mathrm{mL})(n=6)$. All results are presented as mean \pm SD. ${ }^{*} p \leq 0.05,{ }^{* *} p \leq 0.01$. NS, non-stimulated; NT, non-treated.

To validate the differences in transcriptional regulation, we determined the populational T-cell changes by flow cytometry. As expected, UCB-MNC-sEV treatment promoted the differentiation into regulatory T-cells (Treg), while inhibiting effector populations (Figure $2 \mathrm{~m}-\mathrm{o}$ ). In this experiment, Tregs were defined as $\mathrm{CD} 4^{+} \mathrm{CD} 25^{+} \mathrm{CD} 127^{-}$cells, and the results were confirmed using a gating strategy based on the expression of the transcription factor Foxp3 (Figure S2). Importantly, UCB-MNC-sEV's effect was shown to be as powerful as IL-2 for the induction of Treg (Figures $2 \mathrm{~m}$ and S2).

\subsection{UCB-MNC-sEV Reduce the Expression of Psoriasis Markers, in an In Vitro 3D Model}

Considering the results from Figure 2, which evidenced an immunomodulatory effect of UCB-MNC-sEV, particularly focused on the Th17 and Treg response, we decided to evaluate the therapeutic benefit of UCB-MNC-sEV in psoriasis. Using an in vitro 3D model of reconstructed human epidermis, engineered to be histologically and metabolically similar to psoriatic skin, we show that UCB-MNC-sEV treatment significantly reduced 
the expression of the inflammatory mediators IL-6, IL-8, CXCL10, and COX-2, and had a tendential effect on IFNG and TNFA mRNA (Figure 3a-h). Moreover, the expression of psoriasin (S100A7) and beta-defensin-2 (DEFB4), two psoriasis-associated antimicrobial peptides, was also significantly decreased (Figure $3 \mathrm{i}, \mathrm{j}$ ).

a

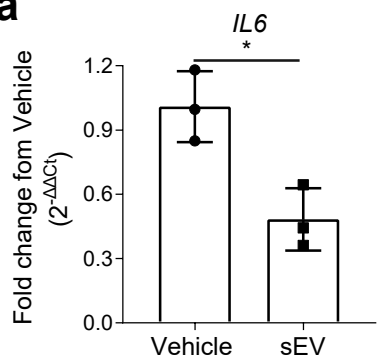

C

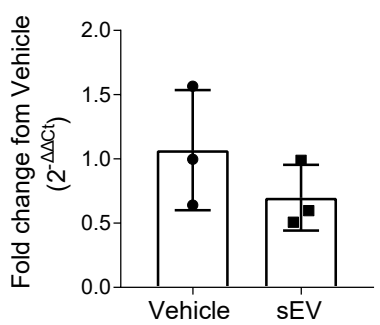

e

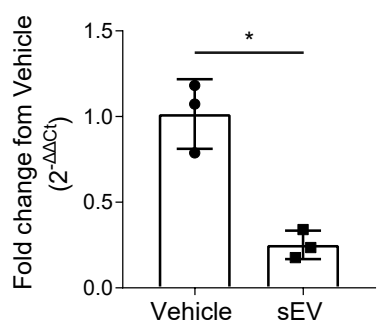

g

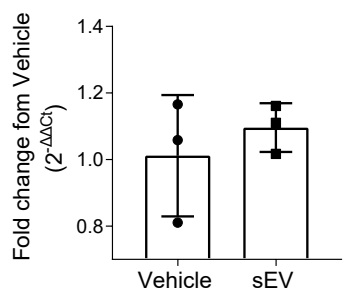

i

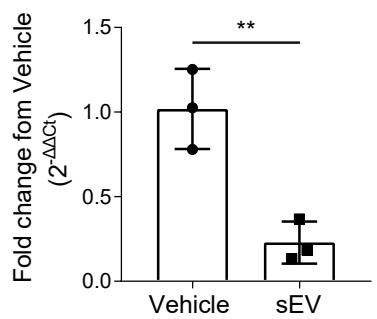

b

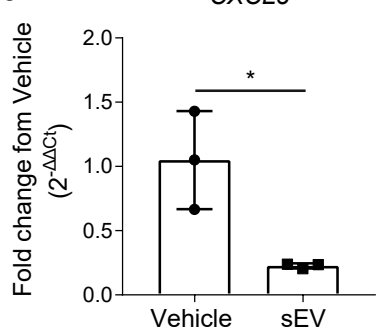

d

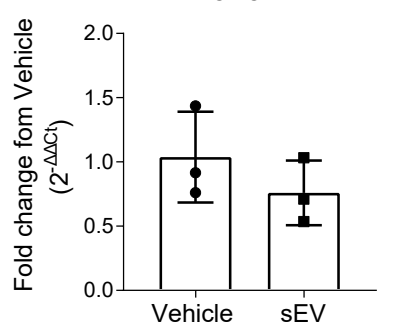

$\mathbf{f}$

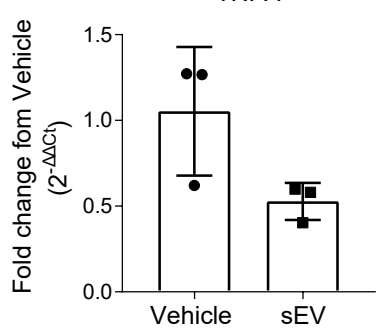

h

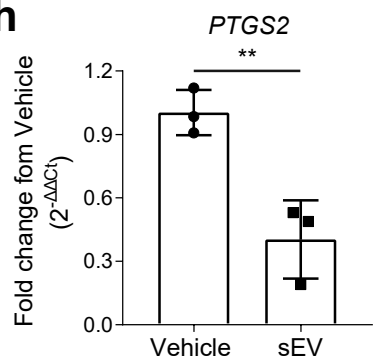

j DEFB4

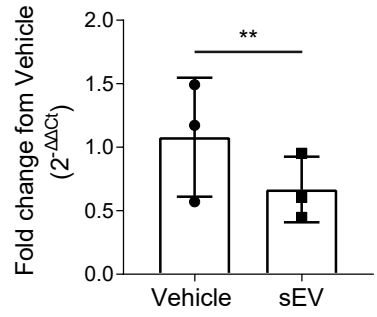

Figure 3. Decreased expression of epidermal psoriatic features after UCB-MNC-sEV treatment. Relative expression of $(\mathbf{a}-\mathbf{h})$ pro-inflammatory mediators and $(\mathbf{i}, \mathbf{j})$ anti-microbial peptides by a 3D model of human psoriatic epidermis, treated with $1 \times 10^{10}$ particles/mL UCB-MNC-sEV twice daily for 6 days $(n=3)$. All results are presented as mean $\pm \mathrm{SD} .{ }^{*} p \leq 0.05,{ }^{* *} p \leq 0.01$. 


\subsection{UCB-MNC-sEV Show a Modest Effect in Imiquimod-Induced Psoriasis, Regulating Keratinocyte Proliferation and T-Cell Homeostasis}

Application of imiquimod, a TLR7 agonist, to mouse skin leads to the development of psoriatic features, such as epidermal thickening, erythema, inflammatory cell infiltration, and epidermal expression of IL-17 [22]. Daily topical treatment with UCB-MNC-sEV, dissolved in a slow-release hydrogel and applied $1 \mathrm{~h}$ after imiquimod, did not significantly ameliorate macroscopic psoriasis-like features, when compared to hydrogel alone (Figure 4a,b). However, UCB-MNC-sEV were significantly superior to hydrogel at reducing acanthosis, as seen by microphotographs (Figure 4c,d). The expression of the inflammatory markers TNF $\alpha$, IFN $\gamma$, IL-17A, CCL20, and CXCL1, albeit significantly reduced when compared to the imiquimod control, was similar between the two groups receiving hydrogel, with or without UCB-MNC-sEV (Figure 4e-i). When comparing the three treatment groups, there were no major changes in the skin infiltration of most inflammatory cells, including neutrophils, macrophages, total T-cells, $\gamma \delta \mathrm{T}$-cells, and total CD4 ${ }^{+} \mathrm{T}-$-cells (Figure $4 \mathrm{j}, \mathrm{k}$ and Figure S3). CD8 ${ }^{+}$T-cells were slightly reduced in UCB-MNC-sEV-treated animals compared to the skin of mice receiving only hydrogel (Figure 4l). Furthermore, UCBMNC-sEV tendentially increased the number of Treg in the skin of imiquimod-treated mice (Figure $4 \mathrm{~m}$ ), a finding that is consistent with gene expression data from diabetic mice with chronic skin wounds (Figure S4) [6,18]. These findings indicate that, in a context of psoriasis, UCB-MNC-sEV improve certain pathological features, possibly through a mechanism that involves both local and immune cells, but did not significantly reduce the overall disease burden in this model. 
a

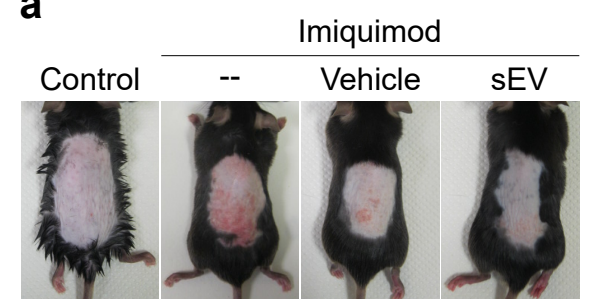

C
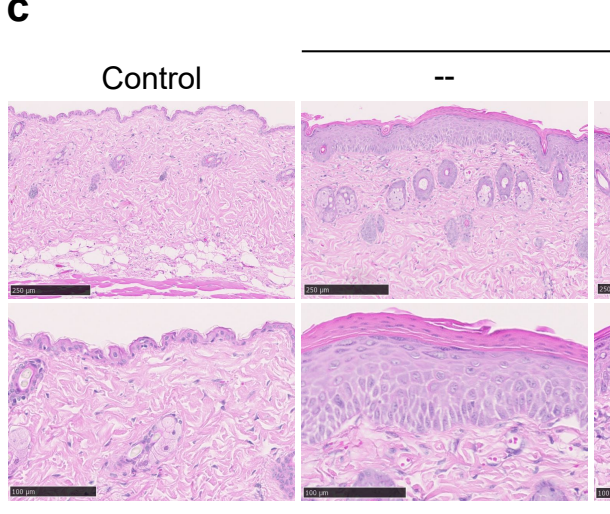

Imiquimod
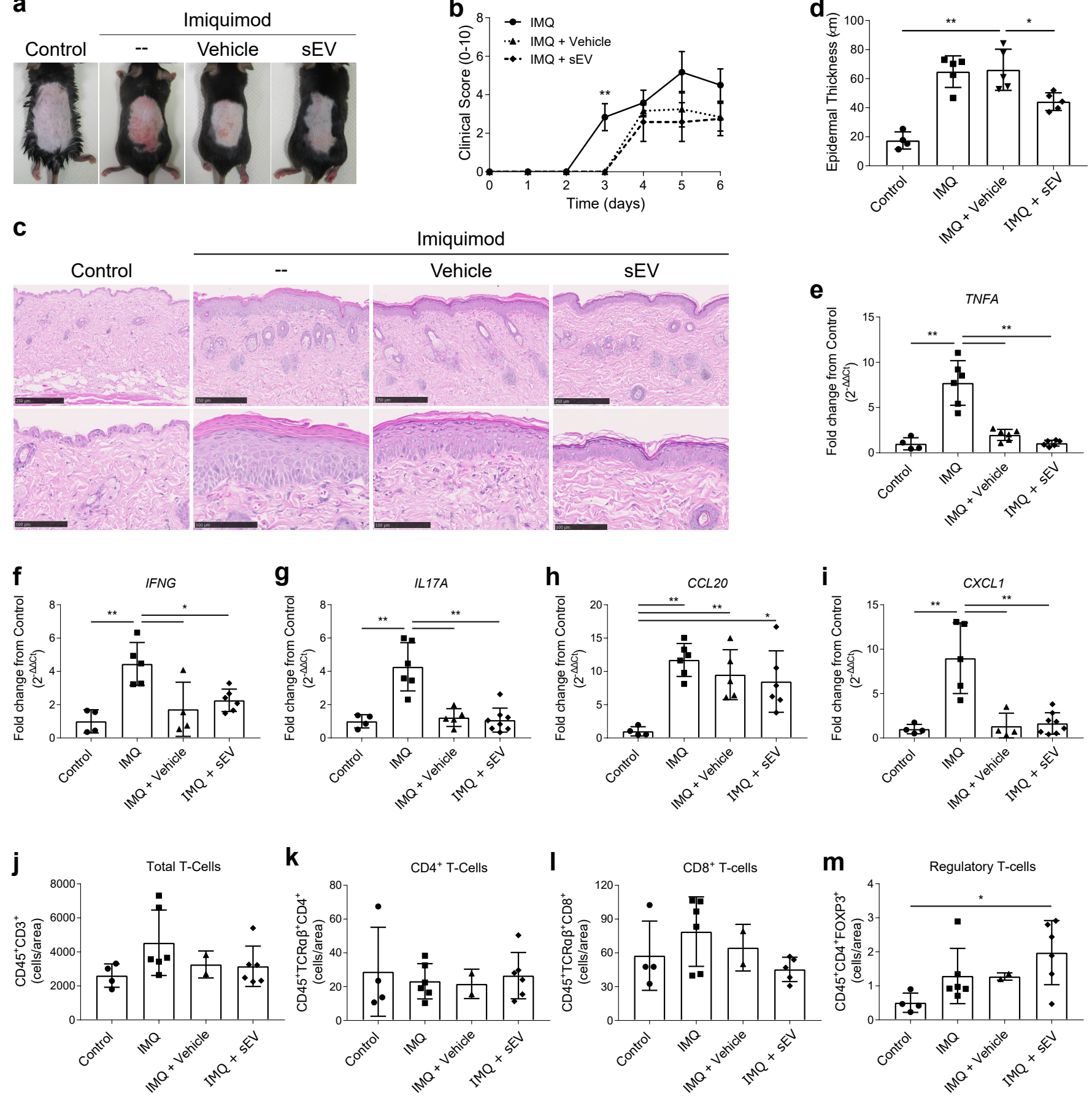

Figure 4. Therapeutic potential of UCB-MNC-sEV for imiquimod-induced psoriasis. C57BL $/ 6$ mice received 5\% imiquimod (IMQ) on their shaved backs (approx. $7 \mathrm{~cm}^{2}$ ), daily for 6 days. A topical formulation containing $2 \times 10^{10}$ particles $/ \mathrm{mL}$ UCB-MNC-sEV was applied to the same area every day, $1 \mathrm{~h}$ after IMQ. (a) Representative photos and (b) clinical score $(n=6)$. (c) Representative H\&E microphotographs used to measure (d) epidermal thickness $(n \geq 4)$. Scale bars $=250 \mu \mathrm{m}$ or $100 \mu \mathrm{m}$, respectively, for upper and lower microphotographs. Epidermal thickness was measured from stratum basale to stratum granulosum, averaging 5 measurements per section, for a total of 20 data points per animal. (e-i) Relative expression of pro-inflammatory mediators in the skin of all tested groups $(n 4)$. (j-m) Digested skin of all four test groups was analyzed by flow cytometry for identification of total, CD4+, CD8+ and regulatory T-cells $(n \geq 2)$. All results are presented as mean \pm SD. ${ }^{*} p \leq 0.05,{ }^{* *} p \leq 0.01$. 


\section{Discussion}

Over the last years, sEV have been explored for their potential as cell-free immunomodulatory and regenerative agents. Indeed, unmodified or engineered sEV were shown to have therapeutic potential across multiple conditions, including cancer [23], inflammatory lung diseases [24,25], and autoimmunity [26]. Here, we explore the mechanism of action of sEV isolated from UCB-MNC, and evaluate their effect in psoriasis models.

In vitro, UCB-MNC-sEV exhibit anti-inflammatory properties, affecting macrophage differentiation and cytokine production. These results are consistent with previous findings using sEV isolated from bone marrow [20] or cord blood [21]. We also show that the presence of UCB-MNC-sEV-induced M2 macrophages has a down-stream effect on neighboring cells, such as skin fibroblasts, reducing their response to an inflammatory trigger. Moreover, UCB-MNC-sEV strongly inhibited cell proliferation and cytokine production by LPS-stimulated total $\mathrm{CD}^{+}$and $\mathrm{CD}^{+} \mathrm{T}$-cells, consistent with previous reports [11,27]. This outcome is possibly due to an effect on T-cell differentiation, given that UCB-MNCsEV promote a shift from a Th1 or Th17 into a Treg phenotype. Notably, UCB-MNC-sEV stimulus was shown to be as effective as IL-2 in promoting Treg development.

Our in vitro findings evidenced a potential mechanism of action for UCB-MNC$\mathrm{sEV}$, responsible for a shift in the expression of transcription factors, which favor Treg differentiation and concomitantly silence Th17 signaling. Biologics targeting the Th17 axis ( $\alpha$-IL-17 and $\alpha$-IL-23) have been proved to be clinically effective in ameliorating psoriasis symptoms [28-30]. Additionally, previous reports suggest that Treg, a typically tolerogenic cell population, play a crucial role in the maintenance of skin homeostasis. Treg-deficient animals present an exacerbated response to imiquimod [31] and Treg from psoriatic patients display an impaired suppressive function [32]. Hence, we hypothesized that UCB-MNCsEV's profile could be therapeutically beneficial in psoriasis. To test this, we employed a model of reconstructed human epidermis, composed of keratinocytes in various stages of differentiation, and pre-treated to display psoriasis-like inflammatory features. UCBMNC-sEV treatment significantly reduced the expression of psoriasis-associated molecules, including IL-6 and IL-8, as well as antimicrobial peptides S100A7 and DEFB4, thereby supporting its therapeutic potential for this disease.

In order to test UCB-MNC-sEV's effect in vivo, we first designed a micelle-rich hydrogel that solidifies at normal body temperature, thus reducing product loss when applied to the skin. Psoriasis-like symptoms were induced by topical applications of imiquimod, and hydrogel was applied $1 \mathrm{~h}$ later, alone or containing UCB-MNC-sEV. In this in vivo model, UCB-MNC-sEV proved superior to hydrogel in reducing or preventing keratinocyte hyperproliferation, as measured by epidermal thickness. Yet, there were no significant differences in the disease scores, mRNA expression and cellular profile between the two treatment groups. While it is possible that the application of hydrogel alone strongly improves psoriatic symptoms, the results observed are better explained by the possible trapping of imiquimod molecules in hydrogel micelles, therefore preventing full symptom development. An alternative experimental setting would either allow for a longer time interval between imiquimod and test treatment applications and/or require the increase of imiquimod dosage. Nevertheless, in line with previous in vitro data, UCB-MNC-sEV had a positive effect on keratinocytes and were tendentially stronger than hydrogel alone in shifting skin cellular infiltrates toward a tolerogenic profile. Importantly, gene expression data from chronic wounds support the increase in Treg differentiation following UCB-MNC-sEV treatment. Given the incomplete therapeutic response of imiquimod-treated animals to UCB-MNC-sEV, it is plausible that UCB-MNC-sEV could act as an adjuvant treatment, in combination with standard therapies, such as anti-IL-17A or anti-IL23. This strategy would not only target immune-driven disease pathways, but also simultaneously stimulate repair mechanisms in the skin.

In conclusion, we show that UCB-MNC-sEV decrease inflammation by targeting different cell populations, such keratinocytes, fibroblasts, and macrophages, and by modulating T-cell differentiation and cytokine production (Figure 5). These findings warrant further 
proof-of-concept studies on the therapeutic potential of UCB-MNC-sEV in inflammatory skin conditions, in particular diseases thought to benefit from Th17/Treg-targeting.

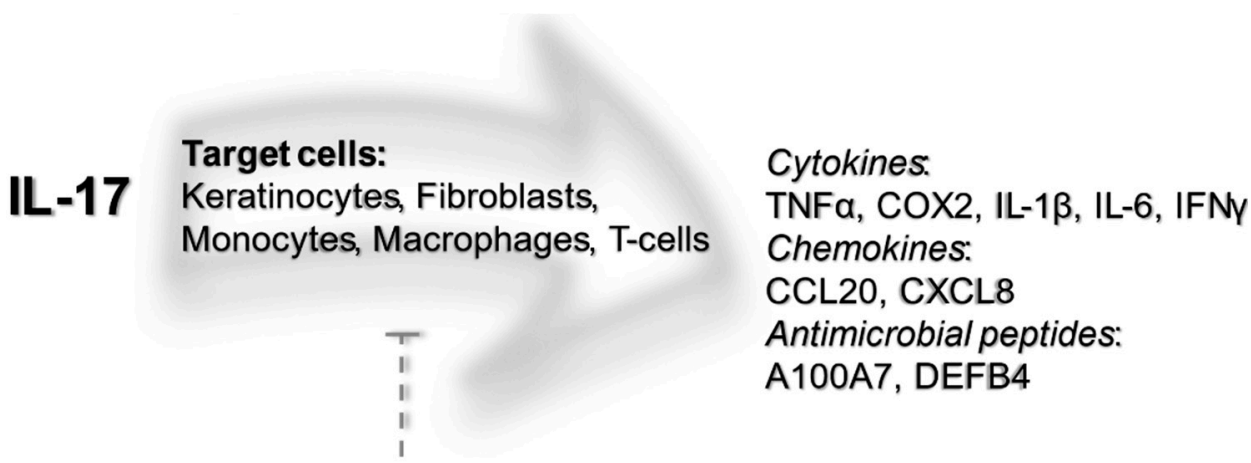

\section{UCB-MNC-SEV}

Figure 5. UCB-MNC-sEV's putative effect on the IL-17 signaling pathway. IL-17, particularly IL-17A, is thought to be a crucial driver of psoriatic disease. IL-17 target cells include keratinocytes, fibroblasts, monocytes, macrophages, and T-cells, all of which are likewise targeted by UCB-MNC-sEV, either directly or indirectly. As shown in this paper, treatment with UBC-MNC-sEV affects multiple players of the IL-17 cascade, and results in a lower expression and/or release of psoriasis-associated mediators, such as TNF $\alpha$, COX2, IL-1 $\beta$, IL-6, IFN $\gamma$, CCL20, CXCL8, A100A7 and DEFB4.

\section{Materials and Methods}

\subsection{UCB Collection, Testing, and Data Protection}

Human UCB samples and relevant donor information were collected following signed informed consent, under approval of the Portuguese National Data Protection Committee and the ethics committees from five Portuguese hospitals, according to local legislation and following the principles of the Declaration of Helsinki. UCB processing, including microbiological testing, and storage were performed by an accredited biobank (Stemlab, S.A, Cantanhede, Portugal).

\subsection{Cell Culture}

\subsubsection{UCB-MNC}

Isolated UCB-MNC were cultured under $0.5 \% \mathrm{O}_{2}$, for $18 \mathrm{~h}$, at a density of 2 million cells $/ \mathrm{mL}$ in serum-free cell culture medium (Lonza AG, Basel, Switzerland) supplemented with $0.5 \mu \mathrm{g} / \mathrm{mL}$ FMS-like tyrosine kinase-3 (Peprotech, London, UK) and $0.5 \mu \mathrm{g} / \mathrm{mL}$ stem-cell factor (Peprotech, London, UK).

\subsubsection{THP-1-Derived Macrophages}

THP-1 cells (ATCC, Manassas, VA, USA) were grown for $72 \mathrm{~h}$ in the presence $25 \mathrm{nM}$ PMA (Sigma, St. Louis, MO, USA). THP-1-derived macrophages were then stimulated for $24 \mathrm{~h}$ with $1 \mu \mathrm{g} / \mathrm{mL}$ LPS (Sigma, St. Louis, MO, USA), followed by a 24-h incubation with $1 \times 10^{10}$ particles $/ \mathrm{mL}$ UCB-MNC-sEV, when indicated.

\subsubsection{Dermal Fibroblasts}

Normal human dermal fibroblasts were kept in Fibroblast Basal Medium, supplemented with Fibroblast Growth Kit Serum-Free, Phenol Red and Penicillin-StreptomycinAmphotericin B Solution (ATCC, Manassas, VA, USA). When appropriate, cells were counted after Hoescht staining (VWR International, Radnor, PA, USA).

\subsubsection{PBMC}

Fresh human PBMC samples were isolated from volunteer donors, following informed consent, by density gradient centrifugation (Stemcell Technologies, Vancouver, Canada). 
For T-cell experiments, $2 \times 10^{5}$ cells/well were activated with PMA (Sigma, St. Louis, MO, USA), anti-CD3/-CD28 (see Table 1 or LPS (Sigma, St. Louis, MO, USA), as indicated, followed by a single dose of UCB-MNC-sEV at $1 \times 10^{10}$ particles $/ \mathrm{mL}$.

Table 1. Human and mouse antibodies for flow cytometry.

\begin{tabular}{|c|c|c|}
\hline Target & Clone & Manufacturer \\
\hline \multicolumn{3}{|l|}{ Anti-human antibodies } \\
\hline CD14 & M5E2 & BD Biosciences, San Jose, CA, USA \\
\hline CD163 & GHI/61 & BD Biosciences, San Jose, CA, USA \\
\hline CD28 & CD28.2 & BioLegend, San Diego, CA, USA \\
\hline CD3 & OKT3 & BioLegend, San Diego, CA, USA \\
\hline CD68 & $\mathrm{Y} 1 / 82 \mathrm{~A}$ & BD Biosciences, San Jose, CA, USA \\
\hline CD86 & FUN-1 & BD Biosciences, San Jose, CA, USA \\
\hline IL-2 & MQ1-17H12 & BioLegend, San Diego, CA, USA \\
\hline \multicolumn{3}{|l|}{ Anti-mouse antibodies } \\
\hline CD11b & $\mathrm{M} 1 / 70$ & BD Biosciences, San Jose, CA, USA \\
\hline CD11c & HL3 & BD Biosciences, San Jose, CA, USA \\
\hline CD16/CD32 & $2.4 \mathrm{G} 2$ & Tonbo Biosciences, San Diego, CA, USA \\
\hline CD25 & PC61.5 & eBioscience, San Diego, CA, USA \\
\hline CD3e & $145-2 \mathrm{C} 11$ & BD Biosciences, San Jose, CA, USA \\
\hline CD4 & RM4-5 & BD Biosciences, San Jose, CA, USA \\
\hline CD45.2 & 104 & eBioscience, San Diego, CA, USA \\
\hline CD62L & MEL-14 & BD Biosciences, San Jose, CA, USA \\
\hline CD8 & $53-6.7$ & BD Biosciences, San Jose, CA, USA \\
\hline Ly6C & AL-21 & BD Biosciences, San Jose, CA, USA \\
\hline Ly6G & $1 \mathrm{~A} 8$ & BD Biosciences, San Jose, CA, USA \\
\hline $\operatorname{TCR} \gamma / \delta$ & GL3 & BioLegend, San Diego, CA, USA \\
\hline $\mathrm{TCR} \alpha / \beta$ & GL2 & BioLegend, San Diego, CA, USA \\
\hline
\end{tabular}

\subsection{UCB-MNC-sEV Isolation}

UCB-MNC culture media were subjected to an optimized isolation process, combining ultrafiltration and size exclusion chromatography [18]. The resulting vesicles were characterized by transmission electron microscopy, flow cytometry, mass spectrometry for protein and lipid composition, RNA sequencing, and nanoparticle tracking analysis, and were found to be rich in CD63 and smaller than $200 \mathrm{~nm}$ [18].

\subsection{Gene Expression}

Total RNA was extracted from cells (THP-1, NHDF, human PBMC, human 3D skin) and tissue (murine skin) with RNeasy Mini Kit (Qiagen, Hilden, Germany) and analyzed with total RNA chips in Bioanalyzer 2100 (Agilent Technologies, Santa Clara, CA, USA). SuperScript ${ }^{\mathrm{TM}}$ IV VILO ${ }^{\mathrm{TM}}$ Master Mix (ThermoFisher Scientific, Waltham, MA, USA) was used for reverse transcription and gene expression was detected by qPCR, using the primer pairs in Table 2.

Table 2. Human and mouse primer sequences for qPCR.

\begin{tabular}{ccc}
\hline Target Gene & Forward Sequence & Reverse Sequence \\
\hline Human & & \\
\hline CXCL1 & & ACTATGGGGGATGCAGGATT \\
CXCL10 & AGGGAATTCACCCCAAGAAC & CTGGATTCAGACATCTCTTCTC \\
DEFB4 & TTCAAGGAGTACCTCTCTCTAG & GAGACCACAGGTGCCAATTT \\
FOXP3 & ATCAGCCATGAGGGTCTTGT & TGGAGGAACTCTGGGAATGT \\
IFNG & GCTTCATCTGTGGCATCATC & CCTTCGCTTGGGCTTAATGA \\
IL1B & CGCCTGCGGGCTCTATC & TTTTCGCTTCCCTGTTTAG \\
& GGTAACTGACTTGAATGTCC & GGTCATTCTCCTGGAAGG \\
\hline
\end{tabular}


Table 2. Cont.

\begin{tabular}{ccc}
\hline Target Gene & Forward Sequence & Reverse Sequence \\
\hline$I L 6$ & GGTACATCCTCGACGGCATCT & GT GCCTCTTTGCTGCTTTCAC \\
$I L 8$ & GTTTTTGAAGAGGGCTGAG & TTTGCTTGAAGTTTCACTGG \\
$P T G S 2$ & ATCTACCCTCCTCAAGTCCC & TACCAGAAGGGCAGGATACAG \\
RORC & TGGACCACCCCCTGCTGAGAA & CTTCAATTTGTGTCTCATGACT \\
S100A7 & CCAAACACACACATCTCACTCA & TCAGCTTGAGTGTTGCTCATC \\
TNFA 21 & GATGTTTGTGGACGTGGTCTTG & CTTTCCACACTGCACCCACTT \\
Mouse & AGGCAGTCAGATCATCTTC & TTATCTCTCAGCTCCACG \\
\hline CCL20 & & \\
FXXP1 3 & & GAGGAGGTTCACAGCCCTTTT \\
$I F N G$ & ACTGTTGCCTCTCGTACATACA & AGAAGCCAGCGTTCACCAGAC \\
IL17A & GCTTGAAGGTGTTGCCCTCAG & GCAAGAGCTCTTGTCCATTGA \\
TNFA & CACCCAGGAAAGACAGCAACC & GTTGCTGATGGCCTGATTGTC \\
\hline
\end{tabular}

\subsection{Protein Quantification and Flow Cytometry (Human)}

TNF $\alpha$ and CCL20 were measured by ELISA (BioLegend, San Diego, CA, USA). M1 macrophages were defined as $\mathrm{CD} 14^{-} \mathrm{CD} 68^{+} \mathrm{CD} 86^{+}$, and $\mathrm{M} 2$ macrophages as $\mathrm{CD} 14^{-} \mathrm{CD} 68^{+}-$ $\mathrm{CD} 163^{+}$by flow cytometry. Tregs were defined as $\mathrm{CD} 4^{+} \mathrm{CD} 25^{+} \mathrm{CD} 127^{-}$cells and the results were confirmed using a gating strategy based on the expression of the transcription factor FOXP3 (Figure S2). Foxp3 expression was analyzed by flow cytometry using the Foxp3/Transcription Factor Staining Buffer Set (eBioscience, San Diego, CA, USA). Whenever mentioned, IL-2 was used as a positive control for Treg development. Antibody details can be found in Table 2 .

\subsection{Reconstructed Psoriatic Human Epidermis}

The 3D model of "psoriasis-like" human epidermis (Sterlab, Vallauris, France) was used according to the manufacturer's instructions. Briefly, epidermal inserts were placed in a 12-well feeder-plate and allowed to equilibrate in the supplied culture medium for one day at $37^{\circ} \mathrm{C}$ and $5 \% \mathrm{CO}_{2}$, before treatment with $1 \times 10^{10}$ particles $/ \mathrm{mL}$ UCB-MNC-sEV, twice a day for 5 consecutive days.

\subsection{UCB-MNC-sEV Formulation for In Vivo Topical Use}

UCB-MNC-sEV are typically formulated in a saline solution. For in vivo experiments, UCB-MNC-sEV were dissolved in a micellar hydrogel, which solidifies at body temperature, thereby reducing product loss when applied to the skin and allowing for a slow particle release (not shown).

\subsection{Imiquimod-Induced Psoriasis}

Animal experiments were approved by the ethical committee of the Spanish National Cardiovascular Research Center, and performed according to national and European regulations, respecting animal welfare guidelines and the 3R's rule. Eight- to twelve-weekold C57BL/ 6 mice received daily applications of imiquimod (3M Pharmaceuticals, Saint Paul, MN, USA) on their shaved backs, for 6 consecutive days. One hour after every imiquimod application, $3 \times 10^{9}$ particles $/ \mathrm{cm}^{2}$ UCB-MNC-sEV dissolved in hydrogel were delivered topically. Epidermal thickness was measured on H\&E-stained samples, using the software NanoZoomer Digital Pathology NDP.view2 (Hamamatsu Photonics, Hamamatsu, Japan), from stratum basale to stratum granulosum, averaging 5 measurements per section, for a total of 20 data points per animal. For flow cytometry, skin was digested with $0.25 \mathrm{mg} / \mathrm{mL}$ Liberase (Roche, Basel, Switzerland) in serum-free RPMI, for $60 \mathrm{~min}$ at $37^{\circ} \mathrm{C}$. Skin cell suspensions were stained with fluorescently labelled antibodies, following Fc 
Block (Table 2). Absolute cell counts were performed using Trucount tubes (BD Biosciences, San Jose, CA, USA). RNA analyses were performed as described above.

\subsection{Statistical Analyses}

Data analyses were performed with Prism 6 (GraphPad Software, San Diego, CA, USA). Unpaired t-tests or one-way ANOVA were employed whenever appropriate $(p<0.05)$.

Supplementary Materials: The following are available online at https:/ /www.mdpi.com/article/10 $.3390 /$ ijms22189797/s1.

Author Contributions: Conceptualization, S.C.R., R.M.S.C., F.V.D. and J.S.-C.; methodology, S.C.R., R.M.S.C., C.F.G. and F.V.D.; validation, S.C.R., R.M.S.C., C.F.G., F.V.D.; formal analysis, S.C.R., P.C.F.; investigation, S.C.R., R.M.S.C., C.F.G. and F.V.D.; data curation, S.C.R., R.M.S.C., C.F.G. and F.V.D.; writing—original draft preparation, P.C.F.; writing—review and editing, S.C.R., R.P.d.N. and J.S.-C.; visualization, S.C.R., P.C.F.; supervision, J.S.-C.; project administration, R.M.S.C. and J.S.-C.; funding acquisition, S.C.R., R.M.S.C., C.F.G., F.V.D. and J.S.-C. All authors have read and agreed to the published version of the manuscript.

Funding: This work was co-funded by Regional Operational Program Center 2020, Portugal 2020 and the European Union through ERDF within the scope of project CENTRO-01-0247-FEDER022398 and CENTRO-01-02B7-FEDER-070018. S.C.R.'s work was supported by FCT fellowship SFRH/BD/137633/2018.

Institutional Review Board Statement: The study was conducted according to the guidelines of the Declaration of Helsinki, and approved by the Ethics Committee of the Spanish National Center for Cardiovascular Research (PROEX160/15, valid from June 2015 to September 2020).

Data Availability Statement: For any data or certificate requests, please contact Exogenus Therapeutics, S.A., at team@exogenus-t.com.

Acknowledgments: The authors greatly appreciated the assistance of Francisco Sanchez Madrid and Danay Cibrian for implementing the imiquimod-induced psoriasis mice model.

Conflicts of Interest: S.C.R., R.M.S.C., P.C.F., C.F.G., F.V.D. and J.S.-C. are or were employees of Exogenus Therapeutics, S.A., R.P.d.N. and J.S.-C. are Exogenus Therapeutics' co-founders and shareholders. R.M.S.C. and J.S-C. are inventors of the patent PCT/IB2017/000412 (use of umbilical cord blood derived exosomes for tissue repair) and S.C.R., R.M.S.C. and J.S.-C. are inventors of the patent PCT/IB2019/058462 (compositions comprising small extracellular vesicles derived from umbilical cord blood mononuclear cells with anti-inflammatory and immunomodulatory properties), currently explored by Exogenus Therapeutics, SA. Financial interest is claimed by Exogenus Therapeutics, S.A., which holds a license (PCT/IB2017/000412) and a patent related to this work (PCT/IB2019/058462). The other authors declared no additional conflicts of interest.

\section{References}

1. Bai, L.; Shao, H.; Wang, H.; Zhang, Z.; Su, C.; Dong, L.; Yu, B.; Chen, X.; Li, X.; Zhang, X. Effects of Mesenchymal Stem Cell-Derived Exosomes on Experimental Autoimmune Uveitis. Sci. Rep. 2017, 7, 4323. [CrossRef]

2. Boehncke, W.-H.; Brembilla, N.C. Unmet Needs in the Field of Psoriasis: Pathogenesis and Treatment. Clin. Rev. Allergy Immunol. 2018, 55, 295-311. [CrossRef] [PubMed]

3. Cao, L.; Xu, H.; Wang, G.; Liu, M.; Tian, D.; Yuan, Z. Extracellular vesicles derived from bone marrow mesenchymal stem cells attenuate dextran sodium sulfate-induced ulcerative colitis by promoting M2 macrophage polarization. Int. Immunopharmacol. 2019, 72, 264-274. [CrossRef] [PubMed]

4. Cardoso, R.M.S.; Rodrigues, S.C.; Gomes, C.F.; Duarte, F.V.; Romao, M.; Leal, E.C.; Freire, P.C.; Neves, R.; Simões-Correia, J. Development of an optimized and scalable method for isolation of umbilical cord blood-derived small extracellular vesicles for future clinical use. Stem Cells Transl. Med. 2021, 10, 910-921. [CrossRef]

5. Del Fattore, A.; Luciano, R.; Pascucci, L.; Goffredo, B.M.; Giorda, E.; Scapaticci, M.; Fierabracci, A.; Muraca, M. Immunoregulatory Effects of Mesenchymal Stem Cell-Derived Extracellular Vesicles on T Lymphocytes. Cell Transplant. 2015, 24, $2615-2627$. [CrossRef] [PubMed]

6. Van Der Fits, L.; Mourits, S.; Voerman, J.S.A.; Kant, M.; Boon, L.; Laman, J.D.; Cornelissen, F.; Mus, A.-M.; Florencia, E.; Prens, E.; et al. Imiquimod-Induced Psoriasis-Like Skin Inflammation in Mice Is Mediated via the IL-23/IL-17 Axis. J. Immunol. 2009, 182, 5836-5845. [CrossRef] [PubMed] 
7. Godfrey, W.R.; Spoden, D.J.; Ge, Y.G.; Baker, S.R.; Liu, B.; Levine, B.L.; June, C.H.; Blazar, B.R.; Porter, S.B. Cord blood CD4(+)CD25(+)-derived T regulatory cell lines express FoxP3 protein and manifest potent suppressor function. Blood 2005, 105, 750-758. [CrossRef]

8. Gordon, K.B.; Strober, B.; Lebwohl, M.; Augustin, M.; Blauvelt, A.; Poulin, Y.; Papp, K.A.; Sofen, H.; Puig, L.; Foley, P.; et al. Efficacy and safety of risankizumab in moderate-to-severe plaque psoriasis (UltIMMa-1 and UltIMMa-2): Results from two double-blind, randomised, placebo-controlled and ustekinumab-controlled phase 3 trials. Lancet 2018, 392, 650-661. [CrossRef]

9. Griffiths, C.E.M.; Armstrong, A.W.; Gudjonsson, J.E.; Barker, J.N.W.N. Psoriasis. Lancet 2021, 397, 1301-1315. [CrossRef]

10. Harris, D.T.; Schumacher, M.J.; Locascio, J.; Besencon, F.J.; Olson, G.B.; DeLuca, D.; Shenker, L.; Bard, J.; Boyse, E.A. Phenotypic and functional immaturity of human umbilical cord blood T lymphocytes. Proc. Natl. Acad. Sci. USA 1992, 89, 10006-10010. [CrossRef]

11. Hartwig, T.; Zwicky, P.; Schreiner, B.; Yawalkar, N.; Cheng, P.; Navarini, A.; Dummer, R.; Flatz, L.; Conrad, C.; Schlapbach, C.; et al. Regulatory T Cells Restrain Pathogenic T Helper Cells during Skin Inflammation. Cell Rep. 2018, 25, 3564-3572. [CrossRef]

12. Henriques-Antunes, H.; Cardoso, R.; Zonari, A.; Correia, J.S.; Leal, E.; Jiménez-Balsa, A.; Lino, M.M.; Barradas, A.; Kostic, I.; Gomes, C.; et al. The Kinetics of Small Extracellular Vesicle Delivery Impacts Skin Tissue Regeneration. ACS Nano 2019, 13, 8694-8707. [CrossRef] [PubMed]

13. Hu, W.; Song, X.; Yu, H.; Sun, J.; Zhao, Y. Released Exosomes Contribute to the Immune Modulation of Cord Blood-Derived Stem Cells. Front. Immunol. 2020, 11, 165. [CrossRef] [PubMed]

14. Karimkhani, C.; Dellavalle, R.P.; Coffeng, L.E.; Flohr, C.; Hay, R.J.; Langan, S.; Nsoesie, E.O.; Ferrari, A.; Erskine, H.E.; Silverberg, J.I.; et al. Global Skin Disease Morbidity and Mortality: An Update From the Global Burden of Disease Study 2013. JAMA Dermatol. 2017, 153, 406-412. [CrossRef]

15. Kim, S.-H.; Lechman, E.; Bianco, N.; Menon, R.; Keravala, A.; Nash, J.; Mi, Z.; Watkins, S.; Gambotto, A.; Robbins, P.D. Exosomes derived from IL-10-treated dendritic cells can suppress inflammation and collagen-induced arthritis. J. Immunol. 2005, 174, 6440-6448. [CrossRef]

16. Krueger, J.G.; Wharton, K.A.; Schlitt, T.; Suprun, M.; Torene, R.I.; Jiang, X.; Wang, C.Q.; Fuentes-Duculan, J.; Hartmann, N.; Peters, T.; et al. IL-17A inhibition by secukinumab induces early clinical, histopathologic, and molecular resolution of psoriasis. J. Allergy Clin. Immunol. 2019, 144, 750-763. [CrossRef]

17. Lai, P.; Weng, J.; Guo, L.; Chen, X.; Du, X. Novel insights into MSC-EVs therapy for immune diseases. Biomark. Res. 2019, 7, 1-10. [CrossRef] [PubMed]

18. Lim, H.W.; Collins, S.A.; Resneck, J.S.; Bolognia, J.L.; Hodge, J.A.; Rohrer, T.A.; Van Beek, M.J.; Margolis, D.J.; Sober, A.J.; Weinstock, M.A.; et al. The burden of skin disease in the United States. J. Am. Acad. Dermatol. 2017, 76, 958-972. [CrossRef]

19. Ma, D.; Xu, K.; Zhang, G.; Liu, Y.; Gao, J.; Tian, M.; Wei, C.; Li, J.; Zhang, L. Immunomodulatory effect of human umbilical cord mesenchymal stem cells on T lymphocytes in rheumatoid arthritis. Int. Immunopharmacol. 2019, 74, 105687. [CrossRef]

20. McBride, J.D.; Rodriguez-Menocal, L.; Badiavas, E.V. Extracellular Vesicles as Biomarkers and Therapeutics in Dermatology: A Focus on Exosomes. J. Investig. Dermatol. 2017, 137, 1622-1629. [CrossRef]

21. Van Niel, G.; D'Angelo, G.; Raposo, G. Shedding light on the cell biology of extracellular vesicles. Nat. Reviews. Mol. Cell Biol. 2018, 19, 213-228. [CrossRef]

22. Papp, K.A.; Merola, J.F.; Gottlieb, A.B.; Griffiths, C.E.; Cross, N.; Peterson, L.; Cioffi, C.; Blauvelt, A. Dual neutralization of both interleukin 17A and interleukin 17F with bimekizumab in patients with psoriasis: Results from BE ABLE 1, a 12-week randomized, double-blinded, placebo-controlled phase 2b trial. J. Am. Acad. Dermatol. 2018, 79, 277-286. [CrossRef] [PubMed]

23. Phinney, D.G.; Pittenger, M.F. Concise Review: MSC-Derived Exosomes for Cell-Free Therapy. Stem Cells 2017, 35, 851-858. [CrossRef] [PubMed]

24. Raposo, G.; Stoorvogel, W. Extracellular vesicles: Exosomes, microvesicles, and friends. J. Cell Biol. 2013, 200, 373-383. [CrossRef] [PubMed]

25. Rodrigues, S.C.; Cardoso, R.M.S.; Gomes, C.F.; Duarte, F.V.; Freire, P.C.; Neves, R.; Simoes-Correia, J. Toxicological Profile of Umbilical Cord Blood-Derived Small Extracellular Vesicles. Membranes 2021, 11, 647. [CrossRef]

26. Sugiyama, H.; Gyulai, R.; Toichi, E.; Garaczi, E.; Shimada, S.; Stevens, S.R.; McCormick, T.S.; Cooper, K. Dysfunctional blood and target tissue CD4+CD25high regulatory T cells in psoriasis: Mechanism underlying unrestrained pathogenic effector T cell proliferation. J. Immunol. 2005, 174, 164-173. [CrossRef]

27. Di Trapani, M.; Bassi, G.; Midolo, M.; Gatti, A.; Kamga, P.T.; Cassaro, A.; Carusone, R.; Adamo, A.; Krampera, M. Differential and transferable modulatory effects of mesenchymal stromal cell-derived extracellular vesicles on T, B and NK cell functions. Sci. Rep. 2016, 6, 24120. [CrossRef] [PubMed]

28. Wang, W.M.; Wu, C.; Jin, H.Z. Exosomes in chronic inflammatory skin diseases and skin tumors. Exp. Dermatol. 2019, 28, 213-218. [CrossRef]

29. Wang, Z.-Y.; Yan, B.-X.; Zhou, Y.; Chen, X.-Y.; Zhang, J.; Cai, S.-Q.; Zheng, M.; Man, X.-Y. miRNA Profiling of Extracellular Vesicles Reveals Biomarkers for Psoriasis. J. Investig. Dermatol. 2021, 141, 185-189. [CrossRef]

30. Yuan, L.; Liu, Y.; Qu, Y.; Liu, L.; Li, H. Exosomes Derived From MicroRNA-148b-3p-Overexpressing Human Umbilical Cord Mesenchymal Stem Cells Restrain Breast Cancer Progression. Front. Oncol. 2019, 9, 1076. [CrossRef] 
31. Zhang, D.; Lee, H.; Wang, X.; Rai, A.; Groot, M.; Jin, Y. Exosome-Mediated Small RNA Delivery: A Novel Therapeutic Approach for Inflammatory Lung Responses. Mol. Ther. 2018, 26, 2119-2130. [CrossRef] [PubMed]

32. Zhu, Y.-G.; Feng, X.-M.; Abbott, J.; Fang, X.-H.; Hao, Q.; Monsel, A.; Qu, J.-M.; Matthay, M.A.; Lee, J.W. Human mesenchymal stem cell microvesicles for treatment of Escherichia coli endotoxin-induced acute lung injury in mice. Stem Cells 2014, 32, 116-125. [CrossRef] [PubMed] 